\title{
ELECTRIC CURRENTS IN THE ATMOSPHERE OF THE SUN
}

\author{
V.I. ABRAMENKO, S.I. GOPASYUK, M.B. OGIR \\ Crimean Astrophysical Observatory \\ 334413, p/o Nauchny, Crimea, USSR
}

\begin{abstract}
The structure of the magnetic field, proper motions of sunspots and electric currents have been studied and related to the evolution of sunspot groups. Further the height variations of the magnetic fluxes and electric currents in active regions have been explored.
\end{abstract}

\section{Introduction}

The appearance of an active region on the sun and the evolution of the magnetic field is determined mainly by plasma motions in the subphotospheric layers. These motions induce electric currents and hence change the exterior magnetic field.

The vector magnetograph was a new step in the investigations of the structure of solar magnetic fields and electric currents (Stepanov and Severny, 1962; Nikulin, 1967). Using observational data on the total vector of the magnetic field, Severny (1965) discovered electric currents in the atmosphere of the Sun.

The evolution of the magnetic field of an active region is closely associated with the variations of the electric currents, which may lead to different types of plasma instabilities, in particular flares.

\section{The observational data}

We have studied the observational data of the total vector of the magnetic field for two active regions: 21. -26.10 .1968 (longitude $\ell=-29^{\circ}$ to $+37^{\circ}$; latitude $\varphi=16^{\circ} \mathrm{N}$ ) and 8. -14.06 .1969$ $\left(\ell=-23^{\circ}\right.$ to $\left.+52^{\circ} ; \varphi=14^{\circ} \mathrm{S}\right)$. The first active region was rather stable, the second was decaying.

The magnetic field was recorded in the Fe I $\lambda 5250.2 \AA$ line with the vector magnetograph of the Crimean Observatory (Nikulin, 1967). We have calculated the vertical component of the electric current density $j_{z}$ using the transverse components of the observed field.

\section{Global electric currents and vortex motions of the plasma}

To obtain a better picture of the electric current structure and the current-generating part 
of the magnetic field, we have displayed in Abramenko and Gopasyuk (1987) the vector of the observed transverse field $\mathbf{H}_{\perp}$ as two components, one along the vector of the potential transverse field, and the other perpendicular to it. The structure of the potential field was computed from the $\mathrm{H}_{z}$ component by solving a Neumann boundary value problem. The component perpendicular to the potential field depends only on the electric current, which determines the orientation of the observed transverse field vector relative to the potential field (see Figure 1.). The arrows reveal the presence of two vortex structures. One is twisted mainly in the clockwise direction and covers the whole leading part of the active region, while the second is twisted in the anti-clockwise direction. The vortex structure of the field confirms the presence of large-scale electric currents extended over the total areas of the leading and following parts of the active region. The current is flowing upwards in the leading part, downwards in the following part, independent of the sign of the leading polarity, and wherever the position of the active region is (northern or southern hemisphere). The values of the upflowing and downflowing currents are the same, approximately $2 \times 10^{12} \mathrm{~A}$. The equality of the currents in the leading and following parts suggests that the current is closed and quasi-stationary.

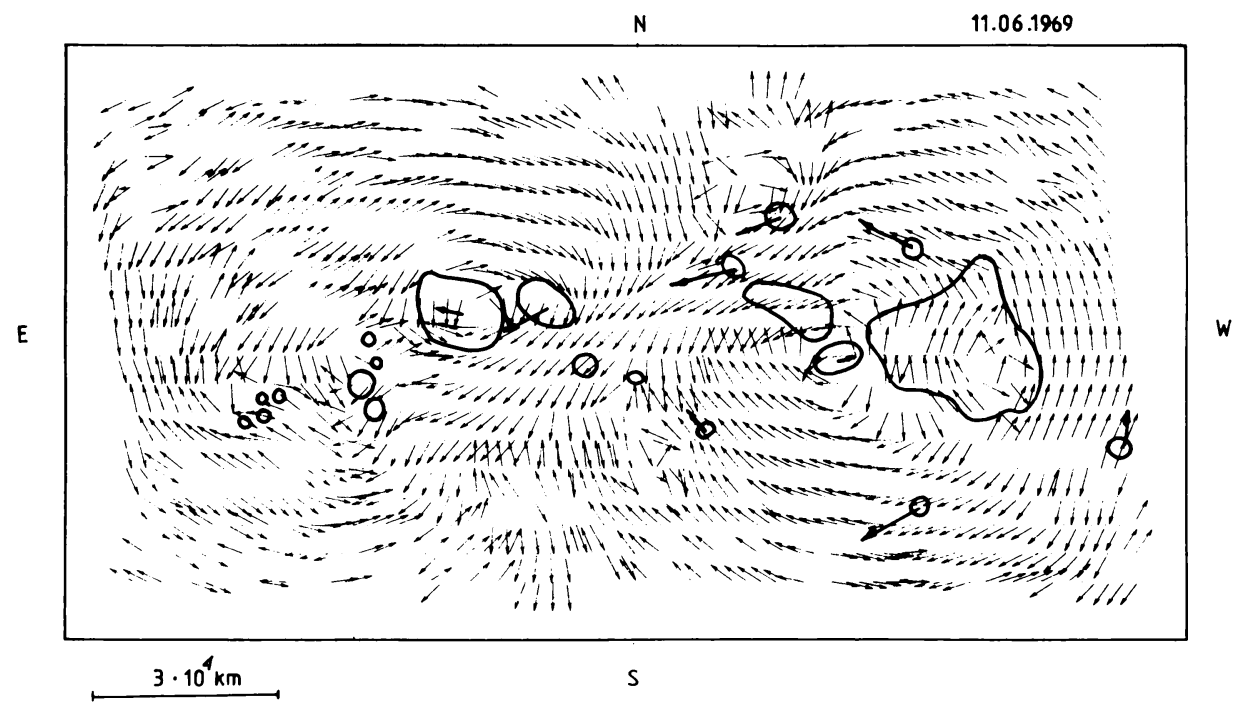

Figure 1. Direction of the azimuth angles (thin arrows) of the component of the observed transverse magnetic field vector that is perpendicular to the transverse field vector computed with the potential approximation. The thick lines show the position of the spots, with their proper motions indicated by thick arrows.

On the maps of the vortex structure of the magnetic field we have plotted the sunspots and indicated their trajectories by arrows. The main spots are located near the centres of the vortex structures. Their proper motions are small. The spots situated at significant distances from the centre of the vortex have large proper motions (up to $200 \mathrm{~m} \mathrm{~s}^{-1}$ ). The trajectories of the spots in general coincide with the direction of the observed component of the transverse field vector that is plotted in Figure 1. This suggests that the proper motion of 
sunspots and the orientation of the non-potential component of the transverse field may have a common origin, both being determined by plasma motions not only at the photospheric level, but also in the subphotospheric layers.

Since in the leading part of the active region the vector of the observed transverse field $\mathrm{H}_{\perp}$ was turning mainly in the clockwise direction and vice-versa in the following part, and the spots were moving along these vortex structures, we have come to the conclusion that there were two plasma vortices in these active regions: one covering the whole leading part, the other covering the following part of the active region. The rotation of the plasma in each of these vortices corresponded to the direction of the magnetic field of the global electric current (Abramenko et al., $1988 \mathrm{c}$ ). The vortex motions of the plasma occupied a rather extensive region from the level of spot formation in the deep layers upward to the photosphere. However, as follows from Figure 1, there exist several regions where the direction of the nonpotential field component does not coincide with the main direction of the vortex. Probably this is a consequence of the circumstance that local disturbances are superposed on the global vortex structure. Our results indicate the presence of processes that twist the whole system of magnetic structures which form an active region.

\section{Local electric currents and the evolution of spot groups}

A system of local small-scale current structures are superposed on the global electric current. Using each individual record, we have calculated the average over the area of the active region of the local electric current density $\bar{j}_{z}$ in the upward and downward directions.

The calculations were made for both sunspot groups. Though these groups were at different stages of evolution, their data appeared to be aligned along the same straight line. The current density $\bar{j}_{z}\left(\mathrm{~A} \mathrm{~km}^{-2}\right)$ and the area $S$ of the group (in square degrees) turned out to be connected by the relation (Abramenko et al., 1987)

$$
\bar{j}_{z}=74 S+950 \text {. }
$$

The correlation coefficient between $\bar{j}_{z}$ and $S$ is 0.88 . This relation shows that the spots in an active region appear only when the average current density exceeds some threshold value, approximately $10^{3} \mathrm{~A} \mathrm{~km}^{-2}$. Furthermore, with increasing area of the spots, the density of the current in the active region changes in synchrony.

From the maps of the observed field we can calculate the energy of the transverse magnetic field of the active region corresponding to a volume with the height of unity, $W=$ $1 / 8 \pi \int_{S} H_{\perp}^{2} \mathrm{~d} S$, and the value of the total current $I_{z}$ (the sum of the local currents in the active region in the given direction). The day-to-day variations of the value of the observed field energy $W$ show a correlation with the variations of the total current $I_{z}$ in the active region (Abramenko et al., 1989). The observational data of both active regions gave a common dependence, which is approximately linear. It is not excluded, however, that each active region has its own dependence. This relation indicates that at least a considerable part of the photospheric transverse field is determined by currents that flow in the photosphere. In fact, if only a small part of the transverse field were produced by photospheric electric currents, its variations against the background magnetic field of the active region would be negligible, and would not have shown a relation with the variations of the photospheric electric currents.

The storage of energy due to the local electric currents in the active region significantly exceeds $10^{32}$ erg (Abramenko et al., 1989). The decay of the active region leads to dissipation 
of the local currents, and the field generally tends to become more potential (Abramenko et al., 1988 a).

\section{Variations of the electric current and magnetic field with height}

We have calculated the electric currents using the structure of the transverse potential field and the orientation of the $\mathrm{H} \alpha$ fibrils (Abramenko et al., $1988 \mathrm{~b}$ ). To avoid projection effects on the $\mathrm{H} \alpha$ fibrils, we have used observations near the central meridian. The calculated currents nicely display not only the structure, but also the evolution of the currents in the photosphere of the active region. This enables us to obtain information concerning the currents not only in the photosphere, but also at the level of the $\mathrm{H} \alpha$ fibrils.

The structure of the potential field was calculated at different levels in the atmosphere up to $50^{\prime} 000 \mathrm{~km}$. Assuming that the deviation of the real transverse field vector from the potential one does not change with height, and is given by the orientation of the $\mathrm{H} \alpha$ fibrils, we have calculated the currents at different levels. The data analysis showed that when going from the photosphere upwards, the main current structures on the whole remain unchanged, and can be observed during at least three days.

The magnetic fluxes were calculated at the same elevations by using the vertical component of the potential field. To compare the height variations of the magnetic flux $\Phi_{z}$ and total electric current $I_{z}$ observed on different days with each other, we normalized them to their values in the photosphere. The photospheric value of the total current was computed using the transverse component of the potential field and the angular deviation of the potential field from the observed transverse field in the photosphere. The results of these calculations for three days of observations are illustrated in Figure 2. We see that the total electric current decreases faster than the magnetic flux of the potential field (Abramenko et al., 1988 b).

A potential model gives the most rapid decrease of the field with height as compared with all other models. The circumstance that the total current decreases much faster than the magnetic flux indicates that the magnetic structures tend to become more potential with height. This agrees with the results reported by Poletto et al. (1975) that the morphology of the coronal structures observed in soft X-ray, and the magnetic field structures computed from the observed $\mathrm{H}_{z}$ component in the photosphere assuming a potential field, look very similar.

\section{Conclusion}

The study of electric currents provides us with exceptional information about the processes taking place in active regions. The currents largely determine the structure and value of the transverse magnetic field at the photospheric level, as well as the evolution of the active region. However, when going to the upper levels of the solar atmosphere, the value of the electric current decreases significantly faster than the magnetic flux. Therefore, if at some level in the corona electric currents are present, they are most likely determined by transient processes associated with different deformations of the coronal magnetic field. 


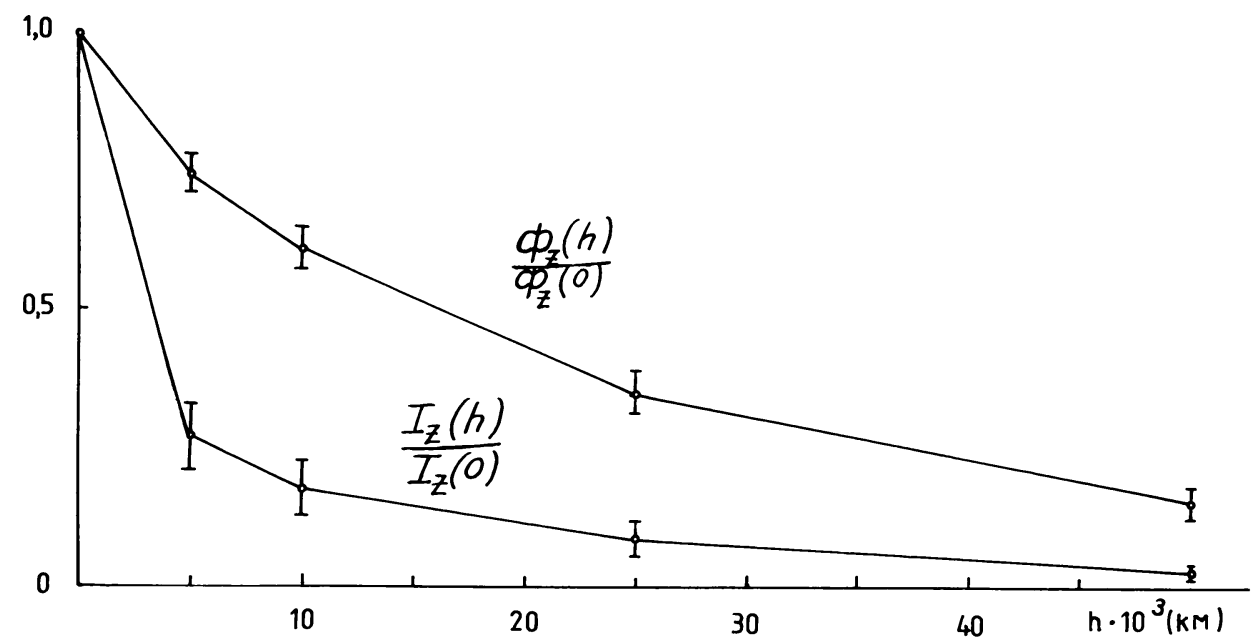

Figure 2. Relative height variations of the magnetic flux and electric current.

\section{References}

Abramenko, V.I. and Gopasyuk, S.I. (1987) 'A system of electric currents and magnetic field structure in the active region', Izv. Krymsk. Astrofiz. Obs. 76, 147-168.

Abramenko, V.I., Gopasyuk, S.I., and Ogir, M.B. (1987) 'Electric currents and active region evolution', Solnech. Dannye 6, 73-79.

Abramenko, V.I., Gopasyuk, S.I., and Ogir, M.B. (1988 a) 'Evolution of the active region, its current systems and flare activity', Izv. Krymsk. Astrofiz. Obs. 78, 151-171.

Abramenko, V.I., Gopasyuk, S.I., and Ogir, M.B. (1988 b) 'The determination of the electric currents regarding the vertical component of the magnetic field and $\mathrm{H} \alpha$ fibrils', Izv. Krymsk. Astrofiz. Obs. 80, 97-105.

Abramenko, V.I., Gopasyuk, S.I., and Ogir, M.B. (1988 c) 'Plasma motions and electric currents in the active region', Izv. Krymsk. Astrofiz. Obs. 81.

Abramenko, V.I., Gopasyuk, S.I., and Ogir, M.B. (1989) 'Electric currents and mangetic field loop structures of the active regions on the Sun', Izv. Krymsk. Astrofiz. Obs. 82.

Nikulin, N.S. (1967) 'Simultaneous registration of the main parameters of a magnetic field by means of a magnetograph', Izv. Krymsk. Astrofiz. Obs. 36, 76-86.

Poletto, G., Vaiana, G.S., Zombeck, M.V., Krieger, A.S., and Timothy, A.F. (1975) 'A comparison of coronal $\mathrm{X}$-ray structures of active regions with magnetic fields computed from photospheric observations', Solar Phys. 44, 83-99.

Severny, A.B. (1965) 'A study of the magnetic field and electric currents of unipolar sunspots', Izv. Krymsk. Astrofiz. Obs. 33, 34-79.

Stepanov, V.E. and Severny, A.B. (1962) 'A photoelectric method for measurements of the magnitude and direction of the solar magnetic field', Izv. Krymsk. Astrofiz. Obs. 28, 166-193. 